\title{
Light-triggered liposomal cargo delivery platform incorporating photosensitizers and gold nanoparticles for enhanced singlet oxygen generation and increased cytotoxicity
}

\author{
This article was published in the following Dove Press journal: \\ International Journal of Nanomedicine \\ 2 February 2017 \\ Number of times this article has been viewed
}

\section{Zofia Kautzka \\ Sandhya Clement \\ Ewa M Goldys \\ Wei Deng}

Centre of Excellence for Nanoscale BioPhotonics, Macquarie University, North Ryde, Sydney, NSW, Australia

Correspondence: Wei Deng

Centre of Excellence for Nanoscale BioPhotonics, Macquarie University, Balaclava Road, North Ryde NSW, 2109, Australia

Tel +6I 298509547

Email wei.deng@mq.edu.au

\begin{abstract}
We developed light-triggered liposomes incorporating 3-5 nm hydrophobic gold nanoparticles and Rose Bengal (RB), a well-known photosensitizer used for photodynamic therapy. Singlet oxygen generated by these liposomes with $532 \mathrm{~nm}$ light illumination was characterized for varying the molar ratio of lipids and gold nanoparticles while keeping the amount of RB constant. Gold nanoparticles were found to enhance the singlet oxygen generation rate, with a maximum enhancement factor of 1.75 obtained for the molar ratio of hydrogenated soy L- $\alpha$-phosphatidylcholine:1,2-dioleoyl-sn-glycero-3-phosphoethanolamine$N$-(hexanoylamine):gold of 57:5:17 compared with liposomes loaded with RB alone. The experimental results could be explained by the local electric field enhancement caused by gold nanoparticles. We further assessed cellular cytotoxicity of gold-loaded liposomes by encapsulating an antitumor drug, doxorubicin (Dox); such Dox-loaded liposomes were applied to human colorectal cancer cells (HCT116) and exposed to light. Gold-loaded liposomes containing RB and Dox where Dox release was triggered by light were found to exhibit higher cytotoxicity compared with the liposomes loaded with RB and Dox alone. Our results indicate that goldloaded liposomes incorporating photosensitizers may serve as improved agents in photodynamic therapy and chemotherapy.
\end{abstract}

Keywords: liposomes, Rose Bengal, gold nanoparticles, singlet oxygen generation, photodynamic therapy

\section{Introduction}

Photodynamic therapy (PDT) is a clinically approved cancer therapy that combines visible light and photosensitizers. Due to its advantages such as minimal toxicity of photosensitizers, low invasiveness, and precise targeting, PDT is applicable to the treatment of cancer, infections, and age-related macular degeneration. ${ }^{1}$ The mechanism by which PDT acts is based on the generation of cytotoxic singlet oxygen $\left({ }^{1} \mathrm{O}_{2}\right)$ and other reactive oxygen species (ROS)., ${ }^{2,3}$ Upon light excitation, the light energy absorbed by photosensitizers is transferred to the surrounding oxygen molecules, causing the formation of ${ }^{1} \mathrm{O}_{2}$ and/or other ROS. These may damage the plasma membranes and DNA, eventually resulting in cell death. ${ }^{4-7}$ Therefore, generation of sufficient amounts of ${ }^{1} \mathrm{O}_{2}$ or other ROS in PDT is a key factor for an effective PDT treatment.

There is intense research interest in the use of gold nanomaterials of varying morphologies to enhance the generation of ${ }^{1} \mathrm{O}_{2}$ and other ROS from photosensitizers. Such nanomaterials are characterized by their distinctive surface plasmon resonance 
(SPR) inducing a strong electromagnetic field around nanostructures when illuminated by light at an appropriate wavelength. ${ }^{8,9}$ The efficacy improvements of PDT using gold nanomaterials were reported by several groups who used gold nanocages conjugated with 2-devinyl-2-(1-hexyloxyethyl) pyropheophorbide (HPPH), ${ }^{10}$ gold nanorod core-silica shell incorporating porphyrin molecules, ${ }^{11}$ gold nanoparticle/ $\mathrm{ZnO}$ nanorod hybrids, ${ }^{12}$ and gold nanoparticles conjugated with 5 -aminolevulinic acid (5-ALA), ${ }^{13}$ to name just a few.

It is worth noting that gold nanostructures can also be used to enable the control of drug release from the liposomes. This is because gold nanoparticles convert light absorbed by the SPR into heat via nonradiative processes. ${ }^{14,15}$ This effect enables the control over drug release from the liposomes. Here, the leading approach involves coating of liposomebased delivery platform with gold nanostructures. ${ }^{16-19}$ In this scenario, liposomes illuminated with laser light (NIR lasers in most cases) experience a temperature rise causing a phase transition in the lipids, which induces drug release. In addition, the same gold nanostructures may enable photothermal therapy (PPT) of cancer. For example, Luo et al demonstrated rapid drug release from gold-coated liposomes triggered by light irradiation with an $808 \mathrm{~nm}$ laser and an enhanced antitumor effect. ${ }^{19}$ In addition to coating with gold nanostructures, very small gold nanoparticles encapsulated inside a liposomal bilayer have been shown to trigger the lipid phase transition via the photothermal effect, leading to the release of liposome contents..$^{20,21}$ It should be mentioned that the small size nanoparticles $(<5.5 \mathrm{~nm})$ used in the latter approach are more advantageous for clinical use than the larger nanostructures generally used for liposome coating, because such small sizes are generally compatible with renal clearance..$^{22,23}$

In the publications mentioned earlier, liposomes incorporating very small gold nanoparticles usually require UV irradiation $(250 \mathrm{~nm})$ to trigger photothermal release, resulting in potential phototoxicity to tissues ${ }^{24}$ as well as suffering from limited UV penetration depth. In the present study, we attempted to trigger the content of liposomes by using photosensitizers in combination with gold nanoparticles through a photodynamic mechanism where the photosensitizers generate ${ }^{1} \mathrm{O}_{2}$ and other ROS following light irradiation at a wavelength coinciding with the absorption band of photosensitizers. Since most of the photosensitizers can be activated with visible light irradiation, ${ }^{25}$ this strategy would reduce the phototoxicity of light sources used for content release from liposomes incorporating small gold nanoparticles.

To this aim, we developed gold-loaded liposomes where 3-5 nm gold nanoparticles were loaded inside a liposomal bilayer. Rose Bengal (RB), a photosensitizer for PDT treatment in breast cancer and metastatic melanoma, ${ }^{26}$ was encapsulated inside the cavity of these liposomes (Figure 1). The ${ }^{1} \mathrm{O}_{2}$ generation from $\mathrm{RB}$ in different liposome samples under $532 \mathrm{~nm}$ laser illumination $\left(37.9 \mathrm{~mW} / \mathrm{cm}^{2}\right)$ for different illumination periods $(1,2,3,4,5$, and 6 min) was
A

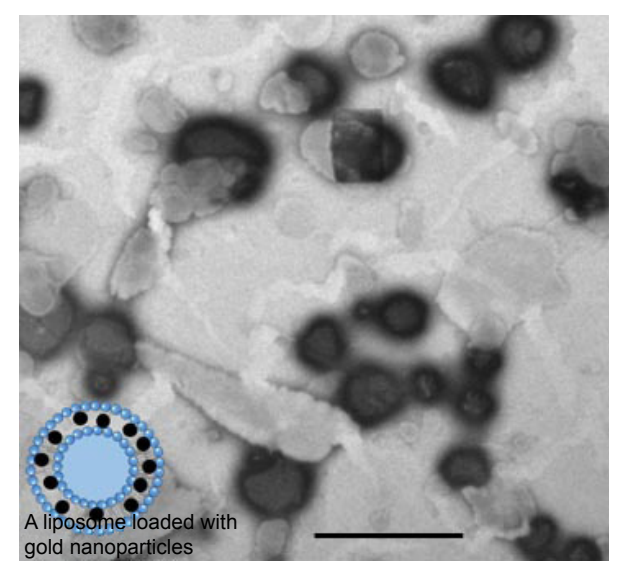

B

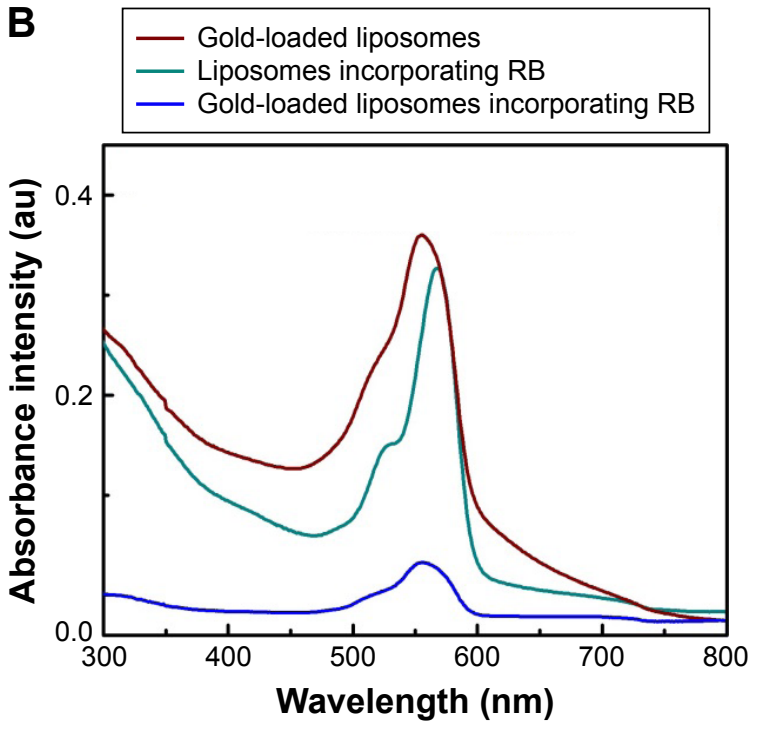

Figure I (A) TEM image of gold-loaded liposomes. Inset is its schematic illustration. The scale bar is 500 nm. (B) Absorption spectra of liposomes incorporating RB alone, gold-loaded liposomes, and liposomes incorporating RB and gold nanoparticles. The molar ratio of HSPC, PE-NH ${ }_{2}$, and gold nanoparticles in gold-loaded liposomes was 57:5:17.

Abbreviations: HSPC, hydrogenated soy L- $\alpha$-phosphatidylcholine; PE-NH ${ }_{2}$, I,2-dioleoyl-sn-glycero-3-phosphoethanolamine- $N$-(hexanoylamine); RB, Rose Bengal; TEM, transmission electron microscopy. 
assessed by using the singlet oxygen sensor green (SOSG). For comparison, the capabilities of ${ }^{1} \mathrm{O}_{2}$ formation using gold-coated liposomes containing RB were also examined under the same experimental conditions. Furthermore, we demonstrated that these gold-loaded liposomes incorporating a mixture of RB and an antitumor drug doxorubicin (Dox) can also release their content when illuminated by light at $532 \mathrm{~nm}$ wavelength. The cytotoxicity assessment in human colorectal cancer cells, HCT116, showed that this was a more effective combination than PDT and standard chemotherapy when applied separately.

\section{Materials and methods Materials}

Hydrogenated soy L- $\alpha$-phosphatidylcholine (HSPC) and 1,2-dioleoyl-sn-glycero-3-phosphoethanolamine- $N$ (hexanoylamine) $\left(\mathrm{PE}-\mathrm{NH}_{2}\right)$ were purchased from Avanti Polar Lipids, Inc. RB, chloroform, sodium hydroxide, gold(III) chloride trihydrate, Triton X-100, hydroxylamine hydrochloride, nitric acid, hydrochloric acid, ammonium sulfate, Dox, and dodecanethiol-functionalized gold nanoparticles were purchased from Sigma-Aldrich. PBS ( $\mathrm{pH}$ 7.4) and fetal bovine serum (FBS) were obtained from Thermo Fisher Scientific. SOSG was supplied from Thermo Fisher Scientific. CellTiter $96^{\circledR}$ AQueous One Solution Cell Proliferation Assay was purchased from Promega (Madison, WI, USA).

\section{Methods}

Preparation of gold-loaded liposomes containing RB

The gold-loaded liposomes were prepared by a coextrusion method with some modification. ${ }^{27}$ The mixture of HSPC, $\mathrm{PE}-\mathrm{NH}_{2}$, and gold nanoparticles at different molar ratios was diluted to $1.0 \mathrm{~mL}$ in total volume using chloroform in a glass test tube, and it was vortexed gently for $10 \mathrm{~min}$. Chloroform was evaporated off with a stream of Argon, and the lipid film was formed around the tube wall. This film was hydrated with $1.0 \mathrm{~mL}$ of RB solution $(0.04 \mathrm{mM})$, followed by vigorous stirring until the suspension was homogenized. The suspension was extruded 11 times in an extruder (Avanti Polar Lipids, Inc) with two $1.0 \mathrm{~mL}$ glass syringes. The pore size of the polycarbonate membrane (Avanti Polar Lipids, Inc) was $100 \mathrm{~nm}$. The resulting suspension was purified by centrifugation $(7,000 \mathrm{rpm}, 10 \mathrm{~min})$ for three times and was stored at $4^{\circ} \mathrm{C}$ for further use.

Preparation of gold-coated liposomes containing RB Gold-coated liposomes used for comparison were synthesized by using a deposition-precipitation protocol. ${ }^{28}$ Briefly,
$200 \mu \mathrm{L}$ of RB-loaded liposome suspension was mixed with $90 \mu \mathrm{L}$ of gold ion solution and diluted to $1.0 \mathrm{~mL}$ in total volume using DI water. The mixture was aged for $10 \mathrm{~min}$ at room temperature, followed by adding $140 \mu \mathrm{L}$ of hydroxylamine hydrochloride $(20 \mathrm{mM})$ as a reducing agent. This produces an abrupt color change of suspension from dark pink to dark purple. The liposome suspension was purified three times by centrifugation at 7,000 rpm for $10 \mathrm{~min}$.

\section{Preparation of gold-liposome nanocomposites containing Dox}

Liposomes loaded with Dox inside the liposomal cavity were prepared by using a gradient exchange method at a mass ratio of 1:10 (drug to lipid) with slight difference. ${ }^{29}$ A total of $1 \mathrm{~mL}$ of ammonium sulfate $(250 \mathrm{mM})$ containing $\mathrm{RB}(0.04 \mathrm{mM})$ was used to hydrate the lipid film. After hydration and extrusion, free ammonium sulfate and $\mathrm{RB}$ were removed by dialysis of liposome suspension against PBS ( $\mathrm{pH} 7.4$ ) with four buffer changes. Dox solution (10 mg/mL) was subsequently added to liposome suspension, followed by incubation for $1 \mathrm{~h}$ at $60^{\circ} \mathrm{C}$. The resulting solution was then dialyzed against PBS ( $\mathrm{pH} 7.4)$ with four buffer changes to eliminate unloaded Dox.

\section{Characterization of gold-liposome nanocomposites}

The extinction spectra of different gold-liposome nanocomposite samples were measured using a spectrophotometer (Cary 5000 UV-Vis-NIR; Varian Inc.). The luminescence spectrum of RB encapsulated inside the liposomes was obtained using a Fluorolog-Tau-3 system from HORIBA Scientific with 450W Xe lamp excitation. Size distribution and zeta potential of liposome samples were measured with a Zetasizer Nano Series from Malvern Instruments. The morphology of liposome-gold nanocomposites was determined using transmission electron microscopy (TEM). The air-dried samples were imaged using a PHILIPS CM10 system at an accelerating voltage of $100 \mathrm{kV}$. Images were captured with an Olympus Megaview G10 camera and iTEM software. To determine the encapsulation efficiency of RB and Dox loaded inside liposomes, Triton X-100 (0.1\%) was added to the as-prepared liposome suspension, resulting in the release of RB and Dox. The RB fluorescence (Ex/Em: 525/564 nm) and Dox fluorescence (Ex/Em: 485/590 nm) was recorded on a Fluorolog-Tau-3 system and compared with the corresponding $\mathrm{RB}$ and Dox standard curves, respectively.

\section{Stability of liposomes (57:5: I7) incorporating RB}

A total of $200 \mu \mathrm{L}$ liposome suspension was diluted in PBS $(\mathrm{pH} 7.4)$ containing $10 \%$ FBS and dialyzed again PBS 
$\left(\mathrm{pH} \mathrm{7.4)}\right.$ for $48 \mathrm{~h}$ at $37^{\circ} \mathrm{C}$. At various time points $(0,2,4$, 18,24 , and $48 \mathrm{~h}$ ), an aliquot of PBS was taken for the fluorescence characterization of the released $\mathrm{RB}$. The total $\mathrm{RB}$ fluorescence was measured by disrupting liposomes with $0.1 \%$ Triton X-100. The percentage of RB release $\left(R_{R B}\right)$ at various time points was calculated as follows:

$$
R_{R B}(\%)=\frac{F_{t}-F_{0}}{F_{\max }-F_{0}} \times 100
$$

where $F_{t}$ and $F_{0}$ indicates the RB fluorescence intensity at various incubation time points and $F_{\max }$ refers to the total RB fluorescence intensity after the disruption of liposomes.

\section{Singlet oxygen generation assays}

SOSG was used to detect singlet oxygen generated from RB loaded inside liposome-gold nanocomposites. The mixture of $5 \mu \mathrm{L}$ SOSG $(0.5 \mathrm{mM})$ and $700 \mu \mathrm{L}$ liposome suspension was placed in a cuvette, and it was irradiated with a $532 \mathrm{~nm}$ continuous wavelength laser $\left(37.9 \mathrm{~mW} / \mathrm{cm}^{2}\right.$, irradiation for $6 \mathrm{~min}$ ). After irradiation, the SOSG fluorescence at $525 \mathrm{~nm}$ upon $488 \mathrm{~nm}$ excitation was recorded using a FluorologTau-3 system.

\section{Cell preparation}

Human colon adenocarcinoma cells (HCT116), human breast cancers (MCF-7) and normal human colon epithelial cells (CCD $841 \mathrm{CoN}$ ) were purchased from the American Type Culture Collection (Rockville, MD, USA). HCT116 cells were cultured in McCoy's 5A (modified) medium; MCF-7 and CCD 841 CoN cells were cultured in Eagle's Minimum Essential Medium (EMEM). All culture media were supplemented with $10 \%$ FBS and $1 \%$ antibiotic-antimycotic. Flasks were maintained in a $37^{\circ} \mathrm{C}$ incubator with $5 \% \mathrm{CO}_{2}$ humidified air. Cells were detached with trypsin and transferred at appropriate dilutions into 96-well plates for cell viability assays.

\section{Studies of cellular cytotoxicity of gold-liposome nanocomposites containing RB and Dox after light illumination}

Before treatment, HCT116 cells $\left(2 \times 10^{4}\right.$ per well) and MCF-7 cells $\left(1 \times 10^{4}\right.$ per well) were respectively grown on $96-$ well plates for $24 \mathrm{~h}$ in culture medium and incubated with liposome samples at different lipid concentrations for $4 \mathrm{~h}$. After old medium was removed and fresh medium was added to cells, all cell groups were irradiated with a $532 \mathrm{~nm}$ laser for $6 \mathrm{~min}\left(14.3 \mathrm{~mW} / \mathrm{cm}^{2}\right)$, except for the group incubated with liposomes loaded with Dox alone. After irradiation, cells were further incubated for another $24 \mathrm{~h}$ prior to cell viability assessment by the MTS assay (Promega). Cell viability was calculated as a percentage of the absorption intensity of the control group, which was set to $100 \%$.

\section{Dark cytotoxicity of gold-loaded liposomes}

CCD 841 CoN cells $\left(2 \times 10^{4}\right.$ per well) were grown on 96-well plates for $24 \mathrm{~h}$ in culture medium. Cells were then incubated with gold-loaded liposomes containing RB and Dox at two concentrations of lipids ( 45 and $4.5 \mu \mathrm{g} / \mathrm{mL}$ ) for $4 \mathrm{~h}$, followed by another $24 \mathrm{~h}$ incubation in fresh medium. The toxicity of gold-loaded liposomes on CCD 841 CoN cells was determined by the MTS test described as earlier.

\section{Results and discussion Characterization of gold-liposome nanocomposites}

The size of gold nanoparticles used in this study was $3-5 \mathrm{~nm}$ (Figure S1). Figure 1A shows the TEM image of goldloaded liposomes where gold nanoparticle clusters were clearly observed due to the higher electron density of gold. Figure 1B shows the absorption spectra of different liposome samples, gold-loaded liposomes, RB-loaded liposomes, and liposomes loaded with gold nanoparticles and RB. Gold nanoparticles in these liposomes are responsible for the SPR band maximum $\sim 556 \mathrm{~nm}$, which largely coincides with the absorption band of RB. The physical parameters of gold-loaded liposomes such as size and zeta potential were also determined (Table 1). The liposomes exhibited negative surface charges lower than $-30 \mathrm{mV}$, indicating high stability in the aqueous solution. In addition, we estimated the encapsulation efficiency of RB and Dox loaded inside liposomes, which was $\sim 88.0$ and $23.4 \%$, respectively. We also calculated the number of gold nanoparticles per goldloaded liposome with a molar ratio (HSPC:PE-NH $\mathrm{N}_{2}$ :gold) of 57:5:17, which was $\sim 109$ as indicated by the ICP-MS data of gold anions in the sample solution. The detailed calculation is provided in the Supplementary materials. The morphology

Table I Mean size, PDI, and zeta potential ( $\zeta$ ) of different liposome samples prepared in this study

\begin{tabular}{llll}
\hline $\begin{array}{l}\text { Gold-loaded liposome } \\
\text { formulations (HSPC:PE-NH }\end{array}$ :gold) & Size $(\mathbf{n m})$ & PDI & $\zeta(\mathbf{m V})$ \\
\hline $57: 5: 8.5$ & $130.1 \pm 1.0$ & 0.096 & $-37.4 \pm 2.5$ \\
$57: 5: 17$ & $124.6 \pm 2.3$ & 0.182 & $-36.8 \pm 2.8$ \\
$57: 5: 34$ & $131.1 \pm 1.3$ & 0.151 & $-35.8 \pm 0.5$ \\
\hline
\end{tabular}

Abbreviations: $\mathrm{HSPC}$, hydrogenated soy L- $\alpha$-phosphatidylcholine; PDI, polydispersity index; $\mathrm{PE}-\mathrm{NH}_{2}$, I,2-dioleoyl-sn-glycero-3-phosphoethanolamine- $\mathrm{N}$-(hexanoylamine). 
and absorption spectrum of the gold-coated liposomes also synthesized in this study for comparison with gold-loaded liposomes are shown in Figure S2. In these gold-coated liposomes, shell-like gold nanostructures coated on the liposomal surface were formed by the interaction between amine group of lipids and gold anions in solution, followed by reduction to gold nanoparticles, which function as nucleation sites for the additional gold to be added on to eventually form a shell. ${ }^{28}$ The SPR band of gold nanostructures in this case was shifted toward the longer wavelength region, with a maximum at $682 \mathrm{~nm}$, reflecting that a strong dependence of the SPR band in gold nanomaterials is on their size and shape. ${ }^{30}$ The cumulative percentage of RB released from liposomes (57:5:17) is shown in Figure S3. Different amounts of RB were released during $48 \mathrm{~h}$ incubation in PBS with 10\% FBS, with $28 \%$ of $\mathrm{RB}$ released at $48 \mathrm{~h}$. It should be mentioned that fast $\mathrm{RB}$ release was observed within $4 \mathrm{~h}$ and followed by sustained release till $48 \mathrm{~h}$. This could be attributed to a burst release of $\mathrm{RB}$ loosely bound to the liposome surface in the beginning.

\section{Evaluation of singlet oxygen generation from gold-liposome nanocomposites in solution}

We assessed ${ }^{1} \mathrm{O}_{2}$ generation from RB loaded inside different gold-loaded liposome formulations by measuring the fluorescence intensity of SOSG in each sample following light illumination. SOSG is a highly selective probe for singlet oxygen detection, showing strong fluorescence signal with a maximum at $525 \mathrm{~nm}$ upon excitation at $488 \mathrm{~nm}$ in the presence of ${ }^{1} \mathrm{O}_{2}{ }^{31}$ The fluorescence enhancement of SOSG as a function of light irradiation time is plotted in Figure 2, which shows different enhancements of ${ }^{1} \mathrm{O}_{2}$ formation depending on the amount of gold nanoparticles used in liposome preparation. The most efficient enhancement was achieved in the liposomes with a molar ratio (HSPC:PE- $\mathrm{NH}_{2}$ :gold) of $57: 5: 17$. Figure $3 \mathrm{~A}$ shows the variation of $\mathrm{SOSG}$ intensity at $525 \mathrm{~nm}$ as a function of laser illumination time from the liposomes loaded with RB alone compared with the liposomes loaded with $\mathrm{RB}$ and gold (HSPC:PE- $\mathrm{NH}_{2}$ : gold =57:5:17). It is possible to calculate the relative singlet oxygen quantum yield of $\mathrm{RB}\left(\varphi_{\mathrm{RB}}\right)$ in liposomes (57:5:17) by using the value of transmittance at $532 \mathrm{~nm}$ calculated from the absorption spectra (Figure 3B) and the following equation: ${ }^{32}$

$$
\varphi_{\mathrm{RB}}=\varphi_{R E F} \frac{\frac{r_{R B}}{\left(1-T_{R B}\right)}}{\frac{r_{R E F}}{\left(1-T_{R E F}\right)}}
$$

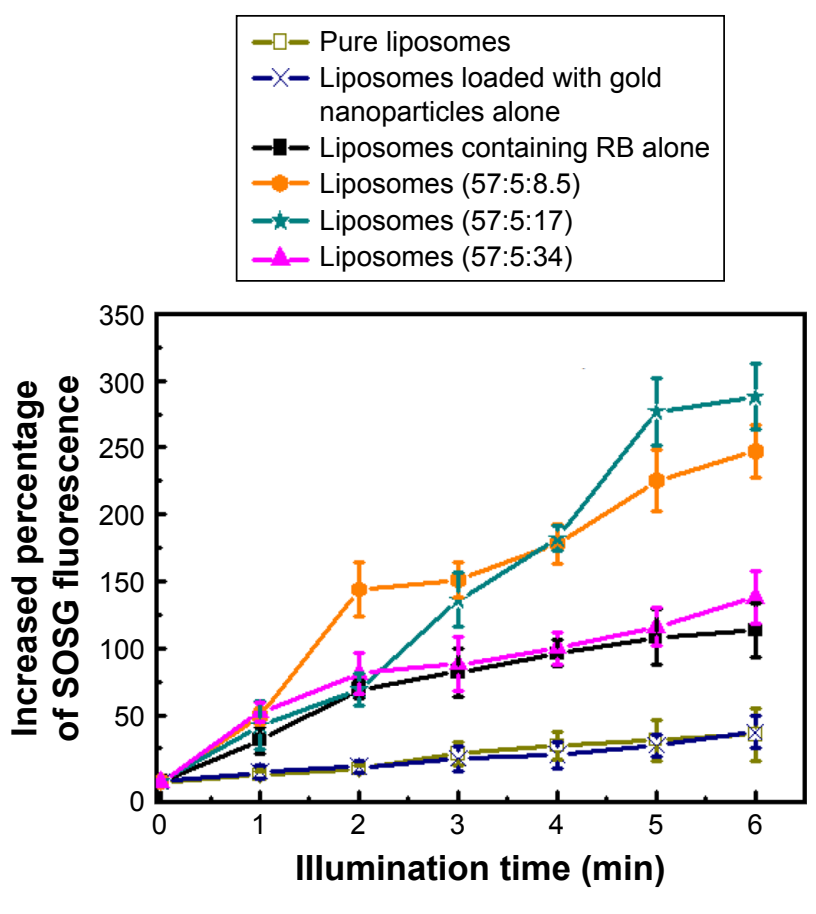

Figure 2 The increased percentage of SOSG fluorescence intensities in various liposome samples exposed to a $532 \mathrm{~nm}$ laser at different illumination time points. Notes: The molar ratio of HSPC, PE- $\mathrm{NH}_{2}$, and gold nanoparticles in gold-loaded liposomes was 57:5:8, 57:5:17, and 57:5:34, respectively.

Abbreviations: HSPC, hydrogenated soy L- $\alpha$-phosphatidylcholine; $\mathrm{PE}-\mathrm{NH}_{2}, \mathrm{I}, 2-$ dioleoyl-sn-glycero-3-phosphoethanolamine- $\mathrm{N}$-(hexanoylamine); RB, Rose Bengal; SOSG, singlet oxygen sensor green.

where $r_{R B}$ and $r_{R E F}$ represent the reaction rates of the fluorescent detection probe with singlet oxygen generated from $\mathrm{RB}$ and a reference photosensitizer respectively. $T_{R B}$ and $T_{R E F}$ indicate the transmittance of RB and the reference photosensitizer at the illumination wavelength, respectively. We estimated $\varphi_{P S}$ of RB in this liposome formulation to be $1.33 \pm 0.27$. This value indicates an enhancement factor of 1.75 compared with liposomes loaded with RB alone. The mechanism of this enhancement is related to the enhanced local field around gold nanoparticles induced by their SPR, which helps to increase the net system absorption. This, in turn, increases the triplet yield of the photosensitizer, subsequently increasing ${ }^{1} \mathrm{O}_{2}$ generation. ${ }^{33,34}$ Similarly, in our previous research on gold-RB conjugates, we also observed the enhancement of ${ }^{1} \mathrm{O}_{2}$ formation when excited at $532 \mathrm{~nm}$ compared with RB alone. ${ }^{32}$ Liposomes with a molar ratio (HSPC:PE- $\mathrm{NH}_{2}$ :gold) of 57:5:8.5 exhibited a relatively efficient ${ }^{1} \mathrm{O}_{2}$ generation with an enhancement factor of 1.53 compared with the liposomes containing RB alone. However, no additional enhancement was observed when increasing the amount of gold nanoparticles (HSPC:PE$\mathrm{NH}_{2}$ : gold $\left.=57: 5: 34\right)$ at the same experimental conditions (Figure 2). Increasing the amount of gold nanoparticles used 

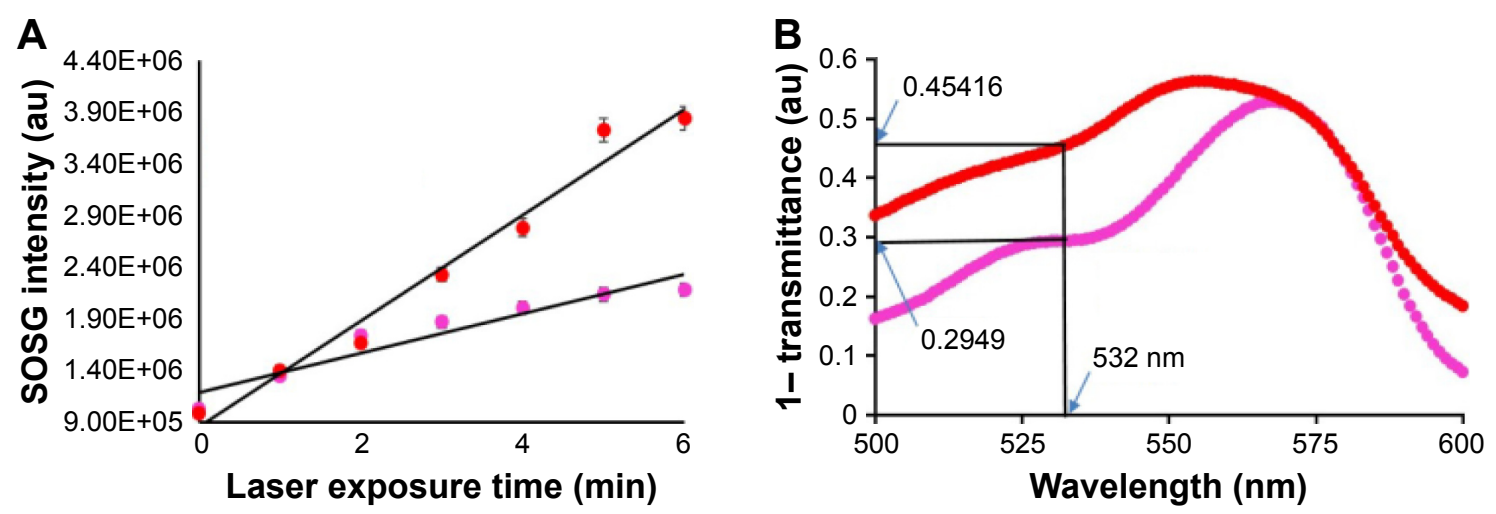

RB alone RB with gold loading

Figure 3 (A) SOSG intensity as a function of laser exposure time for RB alone in liposomes and RB in gold-loaded liposomes. (B) The plot for I- transmittance as a function of wavelength derived from absorption spectra of liposomes containing RB alone and liposomes containing RB and gold.

Abbreviations: RB, Rose Bengal; SOSG, singlet oxygen sensor green.

in the preparation of gold-liposome nanocomposites may increase quenching as well as absorption of fluorescence emitted from SOSG during singlet oxygen generation assays compared with other two liposome formulations (57:5:8.5 and 57:5:17).

For comparison, the assessment of ${ }^{1} \mathrm{O}_{2}$ generation from gold-coated liposomes was also performed under the same experimental conditions. Compared with liposomes loaded with $\mathrm{RB}$ alone, no increase in the amount of ${ }^{1} \mathrm{O}_{2}$ generated from gold-coated liposomes was observed after 6 min illumination at $532 \mathrm{~nm}$ wavelength (Figure S4). This could be attributed to photothermal properties of gold nanoshells coated on the liposomal surface that induced the conversion of absorbed light into heat. ${ }^{16}$

It is well known that gold nanomaterials cause conditiondependent fluorescence enhancement and quenching effects previously demonstrated with various fluorophores. ${ }^{35,36}$ Therefore, we examined the RB fluorescence intensity before and after releasing gold nanoparticles and RB from the liposomes realized by the addition of Triton X-100 to each sample. Interestingly, the RB fluorescence intensity in gold-loaded liposomes was partially quenched compared with free RB molecules released from these liposome-gold formulations after the addition of Triton X-100 (Figure 4A).
A

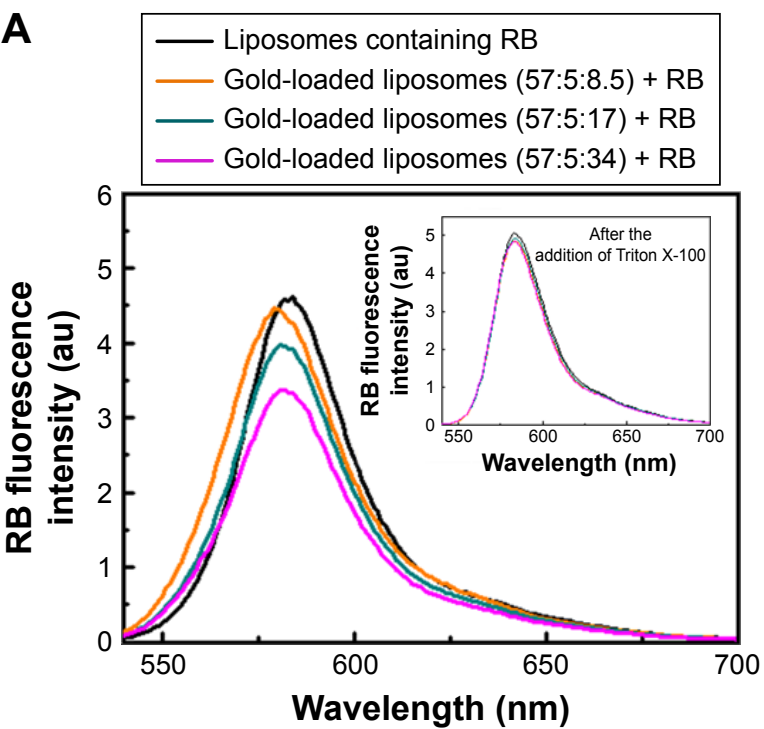

B

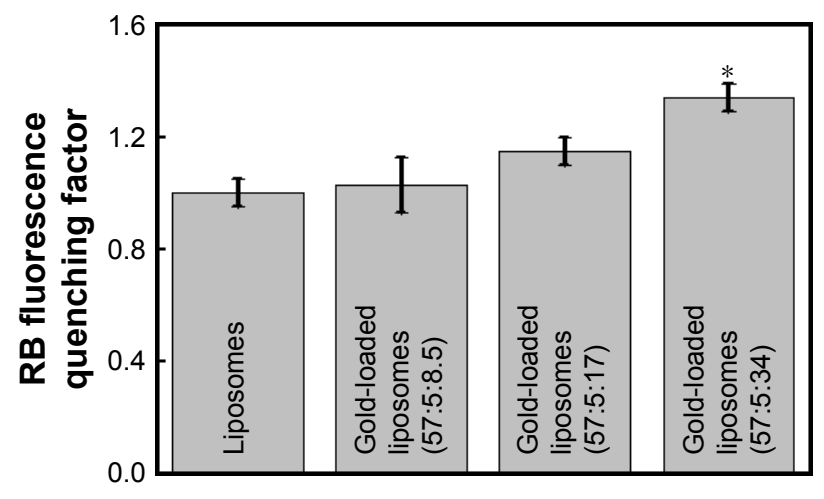

Figure 4 (A) RB fluorescence spectra in pure liposomes and gold-loaded liposomes at $525 \mathrm{~nm}$ excitation wavelength. Inset was the fluorescence spectra of released RB after adding Triton X-100 to each liposome sample. (B) The RB fluorescence quenching factors for different gold-loaded liposomes compared with liposomes containing RB alone. Data points represent the mean \pm SD of three separate experiments $(n=3)$, each performed in triplicate. *P<0.05 compared with liposomes containing RB alone. Abbreviation: RB, Rose Bengal. 
Although the sample with a molar ratio (HSPC:PE-NH 2 :gold) of 57:5:17 quenched the RB fluorescence to some extent (Figure 4B), it exhibited the highest ${ }^{1} \mathrm{O}_{2}$ generation enhancement capability among these gold-loaded liposome samples. Therefore, we chose this sample to further investigate in vitro cell-killing efficacy.

\section{In vitro cellular cytotoxicity and dark cytotoxicity of gold-loaded liposomes containing RB and Dox}

Considering that drugs would be released from liposomes and subsequently execute their action in a light-triggering modality, ${ }^{37,38}$ we encapsulated Dox inside gold-loaded RB-containing liposomes and examined the cytotoxicity of released Dox in HCT116 cells following light illumination. In this way, we expect that combination with chemotherapy and gold nanoparticle-mediated PDT will be able to produce the enhanced cancer cell-killing efficacy. As shown in Figure 5, the cell mortality clearly increased when the cancer cells were treated with gold-loaded liposomes containing RB and Dox (gold-loaded liposomes + RB + Dox) and illuminated with light for $6 \mathrm{~min}$, with a maximum of $38 \%$ cells killed at a lipid concentration of $15.7 \mu \mathrm{g} / \mathrm{mL}$. Their cell-killing efficacy was more efficient than liposomes loaded with RB and Dox

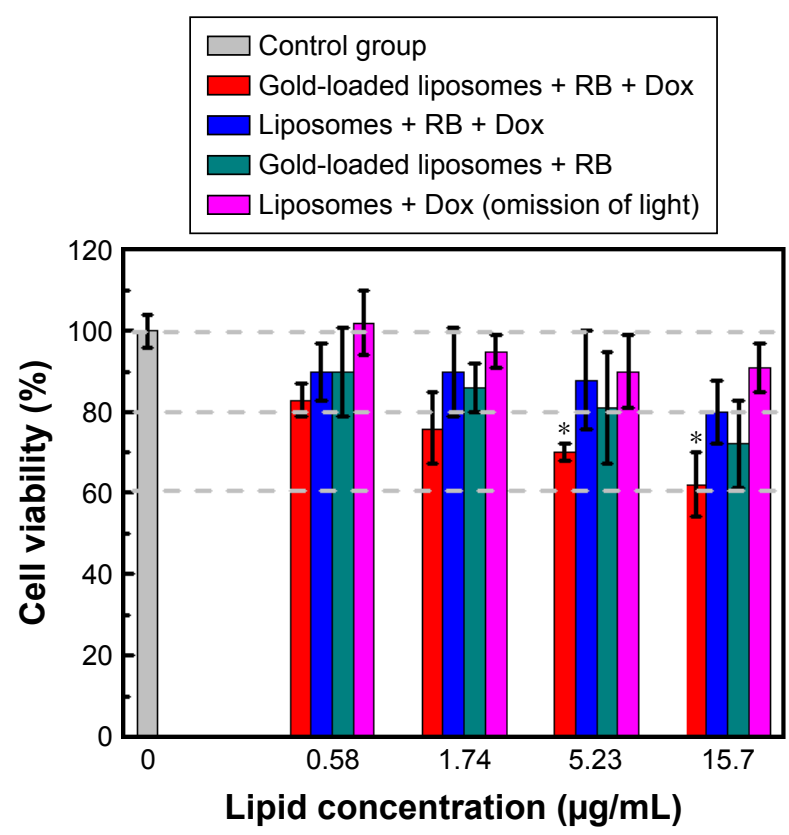

Figure 5 Cytotoxicity of different liposomes with and without gold loading in HCTI 16 cells after 6 min illumination.

Notes: The molar ratio (HSPC:PE- $\mathrm{NH}_{2}$ :gold) of gold-loaded liposome formulation was $57: 5: 17$. Data points represent the mean \pm SD of three separate experiments $(n=3)$, each performed in triplicate. $* P<0.05$ compared with the control group.

Abbreviations: Dox, doxorubicin; HSPC, hydrogenated soy-L- $\alpha$-phosphatidylcholine; $\mathrm{PE}-\mathrm{NH}_{2}, \quad$ I,2-dioleoyl-sn-glycero-3-phosphoethanolamine- $\mathrm{N}$-(hexanoylamine); RB, Rose Bengal. but without gold doping (liposomes + RB + Dox), which induced only $20 \%$ cell death at the same lipid concentration. To confirm that this higher cell-killing efficacy was due to the combination of gold nanoparticle-mediated PDT and chemotherapy, we also separately assessed the cell-killing effect of gold-loaded liposomes containing RB (gold-loaded liposomes + RB) following light illumination and liposomeformulated Dox (liposomes + Dox) without light illumination in cells by varying the concentrations of liposomes. In this case, only $\sim 28$ and $20 \%$ of cell mortality were respectively observed at a lipid concentration of $15.7 \mu \mathrm{g} / \mathrm{mL}$ (Figure 5). In addition to HCT116 cells, we assessed the cell-killing effect of gold-loaded liposomes (gold-loaded liposomes + $\mathrm{RB}+$ Dox) on human breast cancer cell line, MCF-7, with light illumination. Similar to the results on HCT116 cells, the cell viability was also affected (Figure S5). It should be mentioned that the results shown in this study did not represent the optimal conditions for cell killing. The light dose and lipid concentration, in principle, can be increased to achieve the maximum cell killing effect.

Although ${ }^{1} \mathrm{O}_{2}$ generation from gold-coated liposomes containing RB was not observed in our study, there is evidence in the literature that PPT induced by gold nanostructures coated on the liposomal surface can damage cancer cells due to local thermal heating. 17,39,40 Therefore, we examined the cell-killing effect of gold-coated liposomes after laser irradiation. As shown in Figure S6, the cell viability was not obviously changed after treatment with gold-coated liposomes containing RB (gold-coated liposomes + RB) and light illumination. It must be taken into account that PPT requires a good overlap between the absorption band of gold nanostructures and laser excitation wavelength to achieve sufficient photothermal transduction. In our case, the absorption band of gold coating on the liposome surface did not perfectly coincide with excitation light, resulting in minimal energy transformation from light into heat. Similar to the photothermal effect on cell-killing, the heat generated by gold nanostructures after light irradiation also can cause gel-to-liquid phase transition of lipids, triggering drug release from liposomes. Therefore, we evaluated if gold coating can trigger a massive drug release from liposomes upon laser irradiation. This was done by the assessment of cell viability treated with gold-coated liposomes containing RB and Dox (gold-coated liposomes $+\mathrm{RB}+$ Dox) under the same experimental conditions. As shown in Figure S6, there was still no clear increase in cellular cytotoxicity after treatment, indicating negligible leakage of Dox from liposomes after $532 \mathrm{~nm}$ irradiation. As we know, the phase transition temperature of 


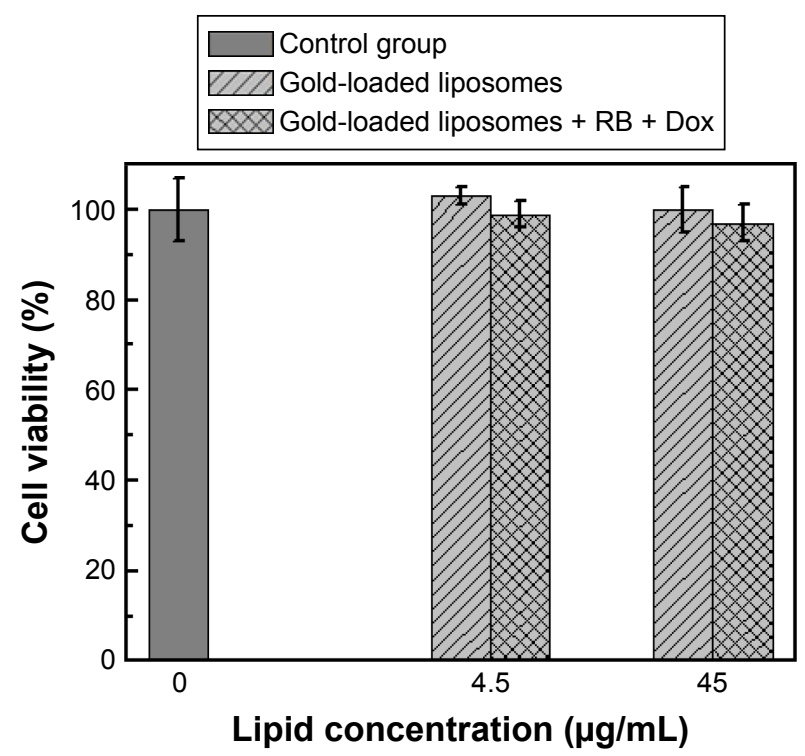

Figure 6 Dark cytotoxicity of gold-loaded liposomes on CCD 84। CoN cells. Notes: The molar ratio (HSPC:PE-NH $\mathrm{N}_{2}$ :gold) of gold-loaded liposomes was 57:5: 17. No significant change was observed by Student's $t$-test.

Abbreviations: Dox, doxorubicin; HSPC, hydrogenated soy L- $\alpha$-phosphatidylcholine; $\mathrm{PE}-\mathrm{NH}_{2}$, I,2-dioleoyl-sn-glycero-3-phosphoethanolamine- $\mathrm{N}$-(hexanoylamine); RB, Rose Bengal.

HSPC and PE-NH $\mathrm{N}_{2}$ used in this study was above $45^{\circ} \mathrm{C},{ }^{41,42}$ which requires sufficient photothermal heat generation from gold nanostructures to trigger lipid phase transition. To this end, we checked if the temperature of gold-coated liposome suspension was increased $>45^{\circ} \mathrm{C}$ after 6 min light illumination and found that it was not obviously changed, indicating that gold nanoshells did not contribute to the generation of sufficient heating for lipid transition and drug release under $532 \mathrm{~nm}$ laser illumination.

The biocompatibility test of gold-loaded liposomes containing $\mathrm{RB}$ and Dox was performed by the evaluation of their dark cytotoxicity on normal human colon cells (CCD $841 \mathrm{CoN}$ ). Cell viability did not show noticeable reduction after the treatment of these liposomes at two concentrations of lipids (45 and $4.5 \mu \mathrm{g} / \mathrm{mL}$ ) compared with the control group (Figure 6). Our results indicate that under in vitro conditions, gold-loaded liposomes are likely not to affect the viability of CCD 841 CoN cells.

\section{Conclusion}

In this study, we developed gold-loaded liposomes containing a photosensitizer RB. We compared their capability for ${ }^{1} \mathrm{O}_{2}$ generation enhancement by changing the amount of gold nanoparticles used in the liposome formulation. A maximum enhancement factor of 1.75 was achieved when the molar ratio of $\mathrm{HSPC}: \mathrm{PE}-\mathrm{NH}_{2}$ :gold was 57:5:17 and illuminated at $532 \mathrm{~nm}$ wavelength for $6 \mathrm{~min}$. We calculated the relative singlet oxygen quantum yield of RB encapsulated in this formulation, which was $1.33 \pm 0.27$. We further assessed cellular cytotoxicity of gold-loaded liposomes containing RB and Dox in HCT116 cells at $532 \mathrm{~nm}$ illumination. They exhibited higher cell-killing efficacy compared with liposomes containing RB and Dox alone under the same experimental conditions. These results indicate that gold nanoparticles encapsulated inside liposomes can contribute to enhanced ${ }^{1} \mathrm{O}_{2}$ generation and the triggered drug release can be induced by this liposome platform with light illumination. The combination of gold nanoparticle-mediated PDT and concurrently triggered chemotherapy may have potential to be used for improved cancer treatment.

\section{Acknowledgments}

All TEM images in this study were taken at the Microscopy Unit, Faculty of Science and Engineering at Macquarie University. We acknowledge PhD student Fei Wang from the Centre of Excellence for Nanoscale BioPhotonics at Macquarie University for supplying us with the cell lines. We also thank Mr Peter Wieland from the Department of Earth and Planetary Science at Macquarie University for his assistance in ICP-MS measurements. This work is supported by Discovery Early Career Researcher Award scheme (DE130100894) and Centre of Excellence scheme (CE140100003) from Australian Research Council.

\section{Disclosure}

The authors report no conflicts of interest in this work.

\section{References}

1. Triesscheijn M, Baas $\mathrm{P}, \mathrm{Schellens} \mathrm{JH}$, et al. Photodynamic therapy in oncology. Oncologist. 2006;11(9):1034-1044.

2. Wang J, Zhang L, Chen M, Gao S, Zhu L. Activatable ferritin nanocomplex for real-time monitoring of caspase-3 activation during photodynamic therapy. ACS Appl Mater Interfaces. 2015;7(41): 23248-23256.

3. Wang G, Zhang F, Tian R, et al. Nanotubes-embedded indocyanine green-hyaluronic acid nanoparticles for photoacoustic-imaging-guided phototherapy. ACS Appl Mater Interfaces. 2016;8(8):5608-5617.

4. Ravanat J-L, Remaud G, Cadet J. Measurement of the main photooxidation products of 2'-deoxyguanosine using chromatographic methods coupled to mass spectrometry. Arch Biochem Biophys. 2000;374(2): 118-127.

5. Wilson BC, Patterson MS. The physics, biophysics and technology of photodynamic therapy. Phys Med Biol. 2008;53(9):R61.

6. Celli JP, Spring BQ, Rizvi I, et al. Imaging and photodynamic therapy: mechanisms, monitoring, and optimization. Chem Rev. 2010;110(5): 2795-2838.

7. Master A, Livingston M, Gupta AS. Photodynamic nanomedicine in the treatment of solid tumors: perspectives and challenges. J Control Release. 2013;168(1):88-102.

8. Dykman L, Khlebtsov N. Gold nanoparticles in biomedical applications: recent advances and perspectives. Chem Soc Rev. 2012;41(6): 2256-2282. 
9. Huang X, Jain PK, El-Sayed IH, El-Sayed MA. Gold nanoparticles: interesting optical properties and recent applications in cancer diagnostics and therapy. Nanomedicine (Lond). 2007;2(5):681-693.

10. Srivatsan A, Jenkins SV, Jeon M, et al. Gold nanocage-photosensitizer conjugates for dual-modal image-guided enhanced photodynamic therapy. Theranostics. 2014;4(2):163-174.

11. Zhao T, Yu K, Li L, et al. Gold nanorod enhanced two-photon excitation fluorescence of photosensitizers for two-photon imaging and photodynamic therapy. ACS Appl Mater Interfaces. 2014;6(4): 2700-2708.

12. Kang Z, Yan X, Zhao L, et al. Gold nanoparticle/ZnO nanorod hybrids for enhanced reactive oxygen species generation and photodynamic therapy. Nano Res. 2015;8(6):2004-2014.

13. Xu H, Yao C, Wang J, Chang Z, Zhang Z. Enhanced 5-aminolevulinic acid-gold nanoparticle conjugate-based photodynamic therapy using pulse laser. Laser Phys Lett. 2015;13(2):025602.

14. Pissuwan D, Valenzuela SM, Cortie MB. Therapeutic possibilities of plasmonically heated gold nanoparticles. Trends Biotechnol. 2006; 24(2):62-67.

15. Gobin AM, Lee MH, Halas NJ, James WD, Drezek RA, West JL. Near-infrared resonant nanoshells for combined optical imaging and photothermal cancer therapy. Nano Lett. 2007;7(7):1929-1934.

16. Oh J, Yoon H-J, Park J-H. Plasmonic liposomes for synergistic photodynamic and photothermal therapy. J Mater Chem B. 2014;2(17): 2592-2597.

17. Rengan AK, Bukhari AB, Pradhan A, et al. In vivo analysis of biodegradable liposome gold nanoparticles as efficient agents for photothermal therapy of cancer. Nano Lett. 2015;15(2):842-848.

18. Troutman TS, Leung SJ, Romanowski M. Light-induced content release from plasmon-resonant liposomes. Adv Mater. 2009;21(22): 2334-2338.

19. Luo L, Bian Y, Liu Y, et al. Combined near infrared photothermal therapy and chemotherapy using gold nanoshells coated liposomes to enhance antitumor effect. Small. 2016;12(30):4103-4112.

20. Paasonen L, Laaksonen T, Johans C, Yliperttula M, Kontturi K, Urtti A Gold nanoparticles enable selective light-induced contents release from liposomes. J Control Release. 2007;122(1):86-93.

21. An X, Zhang F, Zhu Y, Shen W. Photoinduced drug release from thermosensitive AuNPs-liposome using a AuNPs-switch. Chem Commun 2010;46(38):7202-7204

22. Choi HS, Liu W, Misra P, et al. Renal clearance of quantum dots. Nat Biotechnol. 2007;25(10):1165-1170.

23. Zhang X-D, Wu D, Shen X, Liu PX, Fan FY, Fan SJ. In vivo renal clearance, biodistribution, toxicity of gold nanoclusters. Biomaterials. 2012;33(18):4628-4638.

24. Clydesdale GJ, Dandie GW, Muller HK. Ultraviolet light induced injury: immunological and inflammatory effects. Immunol Cell Biol. 2001; 79(6):547-568.

25. DeRosa MC, Crutchley RJ. Photosensitized singlet oxygen and its applications. Coord Chem Rev. 2002;233:351-371.

26. Mousavi SH, Tavakkol-Afshari J, Brook A, Jafari-Anarkooli I. Direct toxicity of Rose Bengal in MCF-7 cell line: role of apoptosis. Food Chem Toxicol. 2009;47(4):855-859.
27. Rasch MR, Rossinyol E, Hueso JL, Goodfellow BW, Arbiol J, Korgel BA Hydrophobic gold nanoparticle self-assembly with phosphatidylcholine lipid: membrane-loaded and janus vesicles. Nano Lett. 2010;10(9): 3733-3739.

28. Kah JC, Phonthammachai N, Wan RCY, et al. Synthesis of gold nanoshells based on the depositionprecipitation process. Gold Bull. 2008; 41(1):23-36

29. Li X, Ding L, Xu Y, Wang Y, Ping Q. Targeted delivery of doxorubicin using stealth liposomes modified with transferrin. Int J Pharm. 2009;373(1):116-123.

30. El-Brolossy T, Abdallah T, Mohamed MB, et al. Shape and size dependence of the surface plasmon resonance of gold nanoparticles studied by photoacoustic technique. Eur Phys J Special Topics. 2008;153(1): 361-364.

31. Shen Y, Lin H, Huang Z, et al. Kinetic analysis of singlet oxygen generation in a living cell using singlet oxygen sensor green. In: Photonics Asia 2010; 2010. Beijing: International Society for Optics and Photonics.

32. Clement S, Sobhan M, Deng W, Camilleri E, Goldys EM. Nanoparticlemediated singlet oxygen generation from photosensitizers. JPhotochem Photobiol A Chem. 2017;332:66-71.

33. Zhang Y, Aslan K, Previte MJR, Geddes CD. Metal-enhanced phosphorescence (MEP). Chem Phys Lett. 2006;427(4):432-437.

34. Zhang Y, Aslan K, Previte MJ, Malyn SN, Geddes CD. Metal-enhanced phosphorescence: interpretation in terms of triplet-coupled radiating plasmons. J Phys Chem B. 2006;110(49):25108-25114.

35. Anger P, Bharadwaj P, Novotny L. Enhancement and quenching of single-molecule fluorescence. Phys Rev Lett. 2006;96(11):113002.

36. Li F, Pei H, Wang L, et al. Nanomaterial-based fluorescent DNA analysis: a comparative study of the quenching effects of graphene oxide, carbon nanotubes, and gold nanoparticles. Adv Funct Mater. 2013; 23(33):4140-4148.

37. Kotova EA, Kuzevanov AV, Pashkovskaya AA, Antonenko YN. Selective permeabilization of lipid membranes by photodynamic action via formation of hydrophobic defects or pre-pores. Biochim Biophys Acta. 2011 1808(9):2252-2257.

38. Tejero I, Gonzalez-Lafont A, Lluch JM, Eriksson LA. Photo-oxidation of lipids by singlet oxygen: a theoretical study. Chem Phys Lett. 2004; 398(4):336-342.

39. Wu C, Yu C, Chu M. A gold nanoshell with a silica inner shell synthesized using liposome templates for doxorubicin loading and nearinfrared photothermal therapy. Int J Nanomed. 2011;6:807.

40. Rengan AK, Jagtap M, De A, Banerjee R, Srivastava R. Multifunctional gold coated thermo-sensitive liposomes for multimodal imaging and photo-thermal therapy of breast cancer cells. Nanoscale. 2014;6(2) 916-923.

41. Chen J, Cheng D, Li J, et al. Influence of lipid composition on the phase transition temperature of liposomes composed of both DPPC and HSPC. Drug Dev Ind Pharm. 2013;39(2):197-204.

42. Kastantin M, Ananthanarayanan B, Karmali P, Ruoslahti E, Tirrell M. Effect of the lipid chain melting transition on the stability of DSPE-PEG (2000) micelles. Langmuir. 2009;25(13):7279-7286.
International Journal of Nanomedicine

\section{Publish your work in this journal}

The International Journal of Nanomedicine is an international, peerreviewed journal focusing on the application of nanotechnology in diagnostics, therapeutics, and drug delivery systems throughou the biomedical field. This journal is indexed on PubMed Central, MedLine, CAS, SciSearch $®$, Current Contents $\AA /$ Clinical Medicine,

\section{Dovepress}

Journal Citation Reports/Science Edition, EMBase, Scopus and the Elsevier Bibliographic databases. The manuscript management system is completely online and includes a very quick and fair peer-review system, which is all easy to use. Visit http://www.dovepress.com/ testimonials.php to read real quotes from published authors. 